\title{
Near Infrared Spectroscopy in Natural Products Analysis
}

Author

Affiliation

\author{
Daniel Cozzolino
}

The Australian Wine Research Institute, Waite Campus, Urrbrae, Australia
Key words

near infrared spectroscopy

- natural products

- chemometrics

- quantitative analysis received July 22,2008

revised November 23, 2008

accepted November 27, 2008

Bibliography

DOI $10.1055 / \mathrm{s}-0028-1112220$

Planta Med 2009; 75: 746-756

(c) Georg Thieme Verlag KG

Stuttgart · New York

Published online January 22, 2009

ISSN 0032-0943

Correspondence

Daniel Cozzolino

The Australian Wine Research Institute

Waite Campus

PO Box 197

Urrbrae - 5064

Australia

Fax: +61-8-8303-6601

daniel.cozzolino@awri.com.au

\section{Abstract}

Several medicinal and herbal plants properties are related to individual compounds such as essential oils, terpenoids, flavonoids, which are present in natural products in low concentrations (e.g., ppm or ppb). For many years, the use of classical separation and chromatographic and spectrometric techniques such as high performance liquid chromatography (HPLC), gas chromatography (GC), liquid chromatography (LC) and mass spectrometry (MS) were initially used for the elucidation of isolated compounds from different plant matrices. Spectroscopic techniques in the infrared (IR) wavelength region of the electromagnetic spectrum have been used in the food industry to monitor and evaluate the composition of foods. Although Herschel discovered light in the near-infrared (NIR) region as early as 1800 , most spectroscopists of the first half of the last century ignored it, in the belief that it lacked any analytical interest. However, during the last 40 years NIR spectroscopy has become one of the most attractive and used methods for analysis. This mini-review highlights recent applications of NIR spectroscopy to the qualitative and quantitative analysis of plant natural products.

\section{Abbreviations}

$\nabla$

ANN: artificial neural network

AOTF: acousto-optic tunable filter

ATR: attenuated total reflection

CP: $\quad$ crude protein

DA: discriminant analysis

DM: dry matter

DOSC: direct orthogonal signal correction

dw: dry weight

FT-NIR: Fourier transform near infrared

GC: gas chromatography
G-G: $\quad$ glycosylated glucose

GSL: glucosinolates

InGaAs: indium gallium arsenide

iPLS: interval partial least squares

IR: infrared

LC: liquid chromatography

LCTF: liquid crystal tunable filters

MEMS: micro electro mechanical systems

MIR: mid infrared

MLR: multiple linear regression

MPLS: modified PLS

MSC: multiplicative scatter correction

MS: $\quad$ mass spectrometry

NIR: near infrared reflectance

NOCH: NIR on combine harvest

PCA: principal component analysis

PDA: photodiode array

PLS: $\quad$ partial least squares

SECV: standard error of cross validation

SEP: $\quad$ standard error of prediction

Si: silicon

siPLS: $\quad$ synergy interval partial least squares

SVM: $\quad$ support vector machine

SIMCA: soft independent modelling of class analogies

$\mathrm{R}: \quad$ correlation coefficient

$\mathrm{R}^{2}$ : $\quad$ coefficient of determination

RPD: $\quad$ residual predictive deviation

RMSEC: root mean square error of calibration

RMSECV: root mean square error of cross validation

RMSEP: root mean square rrror of prediction

REAC: trolox equivalent antioxidant capacity

TLC: $\quad$ thin layer chromatography

THC: tetrahydrocannabinol

UV: $\quad$ ultraviolet

VIS: $\quad$ visible

WT: wavelet transformation 


\section{Introduction}

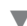

Herbal medicine is the oldest and most widely used form of medicine in the world [1]. Currently, there is increasing interest in medicinal and spice plant species because of their health-promoting properties. Many medicinal and herbal plants properties are related to individual compounds, and their active chemical ingredient in natural products such as essential oils, terpenoids, flavonoids usually are found in low concentrations (e.g., ppm or ppb) [1], [2], [3]. In recent years, several pharmaceutical companies have initiated sophisticated plant screening programmes, applying biochemical high throughput techniques in order to find new drugs with special properties (e.g., anticancer properties) [1], [2], [3].

For many years, the uses of standard separations, chromatographic and spectrometric methods such as high performance liquid chromatography (HPLC), gas chromatography (GC), liquid chromatography (LC) and mass spectrometry (MS) were focused on the elucidation of isolated compounds from different plant matrices [1], [2]. These methods were found useful in phytochemical and plant physiology studies as a fingerprinting method for comparing natural and synthetic samples, and to identify single active compounds [3].

Spectroscopic techniques using the infrared (IR) wavelength region of the electromagnetic spectrum have been used in the food industry to monitor and evaluate the composition and quality value of foods. Although Herschel discovered light in the nearinfrared (NIR) region as early as 1800, many spectroscopists of the first half of the last century ignored it, in the belief that it lacked any analytical interest [4], [5], [6].

NIR spectroscopy is characterised by low molar absorptivities and scattering, which allows nearly effortless evaluation of pure materials. The NIR region of the electromagnetic spectrum, once regarded as having little potential for analytical work, has now become one of the most promising techniques for molecular spectroscopy. The arrival of inexpensive and powerful computers has contributed to the surge of a wide range of NIR applications in several fields, including medical, pharmaceutical, to the most traditional of food analysis [4], [6], [9], [10], [11].

This mini-review highlights recent applications of NIR spectroscopy to the qualitative and quantitative analysis of plant natural products.

\section{Near Infrared Spectroscopy \\ $\nabla$}

Infrared (IR) radiation is the region of the electromagnetic spectrum between the visible (VIS) and the microwave wavelengths [4]. The nominal range of wavelengths for near-infrared is between 750 and $2500 \mathrm{~nm}$ (13400 to $4000 \mathrm{~cm}^{-1}$ ), while for the mid-infrared (MIR), is from 2500 to $25000 \mathrm{~nm} \mathrm{(4000} \mathrm{to}$ $400 \mathrm{~cm}^{-1}$ ) [6], [7], [8]. Solid, liquid or gaseous samples can absorb some of the incoming IR radiation at specific wavelengths resulting in a 'fingerprint' or spectrum [6], [7], [8].

The principle of NIR reflectance spectroscopy is that light of wavelengths between 1100 to $2500 \mathrm{~nm}$, reflected off powdered solids, contains compositional information which can be unravelled by a computer to report multiple analyses almost instantaneously [8], [9], [12]. This method can provide simultaneous, rapid and non-destructive quantitation of major components in many organic substances [8], [9], [12]. Broadly speaking, NIR spectroscopy allows the assessment of organic chemical struc- tures containing $\mathrm{O}-\mathrm{H}, \mathrm{N}-\mathrm{H}$ and $\mathrm{C}-\mathrm{H}$ bonds through the absorption of energy in the NIR region of the spectrum [11]. Spectral 'signatures' in the MIR result from the fundamental stretching, bending, and rotating vibrations of the sample molecules, whilst NIR spectra result from complex overtones and high frequency combinations at shorter wavelengths [4], [5], [6], [8], [12]. Although NIR intensities are 10 - 1000 times lower than for the MIR, highly sensitive spectrometers can be built through several means including the use of efficient detectors and brighter light sources [4], [5], [6], [8]. This allows concentrated bulk or even aqueous materials to be scanned and analysed quickly and easily.

Spectral peaks in the MIR frequencies are often sharper and better resolved than in the NIR domain, the higher overtones (e.g., $1^{\text {st }}$ through $6^{\text {th }}$ ) of the $\mathrm{O}-\mathrm{H}$ (oxygen-hydrogen), N-H (nitrogenhydrogen), $\mathrm{C}-\mathrm{H}$ (carbon-hydrogen) and $\mathrm{S}-\mathrm{H}$ (sulphur-hydrogen) bands from the MIR wavelengths are still observed in the NIR region, although much weaker than the fundamental frequencies in the MIR [4], [9]. In addition. the existence of combination bands (e.g., CO stretch and $\mathrm{NH}$ bend in protein), gives rise to a crowded NIR spectrum with strongly overlapping bands [4], [9]. However, a major disadvantage of this characteristic overlap and complexity has been the difficulty of quantification and interpretation of data originated from NIR spectra [9], [12]. On the other hand, the broad overlapping bands can diminish the need for using a large number of wavelengths in calibration and routine analysis [9].

In recent years, new instrumentation and computer algorithms have taken advantage of this complexity and have made the technique much more powerful and simple to use [4], [9]. The NIR spectrum of an organic material can give a global signature of composition which, with the application of chemometric techniques (e.g., principal component analysis and partial least squares regression), can be used to elucidate particular compositional characteristics in the food matrix not easily detected by targeted chemical analysis [9], [10], [12], [13]. This technique has been used to predict chemical composition in several organic matrixes such as forages, cereals, meat and meat products, beverages, fruits and in a diverse range of plant materials (e.g., crude protein, dry matter, sugars, acidity, alcohol) with high accuracy [4], [5], [6], [8], [9], [10], [11], [12].

\section{Instrumentation}

$\nabla$

Several reports can be found in the literature that review the vast amount of work published related to NIR instrumentation. The purpose of this section is to give an overall introduction to the different available instruments commonly used to analyse natural products.

Spectroscopy combined with chemometric methods has the potential to be a powerful tool for the assessment of chemical composition of any given organic substance [9]. Recent advances in chemometrics software and computing power have greatly enhanced the development of rapid analytical methods based on spectroscopic data and their subsequent application in a wide range of industries [12], [13]. Although the instrumentation may require a large capital outlay (up to 150 thousand U.S. dollars) and can be reasonably complex to calibrate and maintain, simple instruments could be developed for general use [14]. As the technology of spectroscopic instrumentation and chemometrics advances further, the resulting spin-offs may further as- 
sist to define and objectively measure chemical composition in different natural products [12], [13]. An NIR spectrophotometer consists of a light source (e.g., a tungsten halogen lamp), sample presentation accessories, monochromator, detector and optical components such as lenses, collimators, beam splitters, integrating spheres and optical fibers [9], [14].

Basically, two main methods are used to collect the NIR spectra of a given sample, namely reflectance and transmittance. In reflectance (e.g., generally used to analyse solids or powder samples) mode light source and detector are mounted under a specific angle, e.g., $45^{\circ}$, to avoid specular reflection. In transmittance (e.g., generally used to analyse liquid samples) mode the light source is positioned opposite to the detector, while in interactance mode the light source and detector are positioned parallel to each other in such a way that light due to specular reflection cannot directly enter the detector [4], [9], [14], [15]. In both reflectance and transmittance modes integrated spheres may also be used to collect light and increase the signal to noise ratio. Transmission measurements, on the other hand, need very high light intensities which can easily burn the sample surface and alter its spectral properties [14], [15].

Spectrophotometers are conveniently classified according to the type of monochromator. For example in a filter instrument, the monochromator is a wheel holding a number of absorption or interference filters, while its spectral resolution is limited [9], [14], [15]. On the other hand, in a scanning monochromator instrument a grating or a prism is used to separate the individual frequencies of the radiation either entering or leaving the sample. The wavelength divider rotates allowing radiation of the individual wavelengths to subsequently reach the detector [14], [15]. For example, in Fourier transform spectrophotometers, interferometers are used to generate modulated light; while the time domain signal of the light reflected or transmitted by the sample onto the sample can be converted into a spectrum via a fast Fourier transform [4], [9], [14], [15]. Often a Michelson interferometer is used, but also polarisation interferometers can be mounted in some spectrophotometers [4], [15].

Recently, photodiode array (PDA) spectrophotometers have been introduced. They consist of a fixed grating that focuses the dispersed radiation onto an array of silicon ( $\mathrm{Si}, 350-1100 \mathrm{~nm}$ ) or indium gallium arsenide (InGaAs, 1100 - $2500 \mathrm{~nm}$ ) photodiode detectors [9], [14], [15]. There is definitely a shift towards PDA systems because of their high acquisition speed (the integration time is typically $50 \mathrm{~ms}$ but can be as low as a few milliseconds) and the absence of moving parts which enables them to be mounted on online fruit grading lines. Miniaturised, portable and low cost versions (less than 20 thousand U.S. dollars) are available from companies, such as Ocean Optics (Dunedin, FL, USA), Zeiss (Jena, Germany), Oriel (Stratford, CT, USA) and Axsun Technologies (Billerica, MA, USA) [14].

Laser-based systems do not contain a monochromator but have different laser light sources or a tunable laser. Acousto-optic tunable filter (AOTF) instruments use a diffraction-based optical band-pass filter that can be rapidly tuned to pass various wavelengths of light by varying the frequency of an acoustic wave propagating through an anisotropic crystal medium [9], [14], [15].

In recent years, liquid crystal tunable filter (LCTF) instruments have been introduced [14], [15]. They use a birefringent filter to create constructive and destructive interference based on the retardation, in phase between the ordinary and extraordinary light rays passing through a liquid crystal. In this way they act as an interference filter to pass a single wavelength of light [14]. By combining several electronically tunable stages in series, high spectral resolution can be achieved [16]

Micro-electro-mechanical systems (MEMS) combine mechanical parts, sensors, actuators and electronics on a common substrate through the use of micro-fabrication technology. This technology represents a paradigm shift for industrial spectroscopy, and enables a variety of new industrial applications [15], [17]. New spectrophotometers have been made using MEMS and became available in recent years (e.g., Axsun Technologies).

Most applications of NIR spectroscopy which are described in the literature essentially rely on defined measurements. In recent years multi- and hyperspectral imaging systems are being incorporated [14], [15], [17], [18]. In this case multispectral (a few wavelengths) or hyperspectral (a continuous range of wavelengths) imaging systems are required. Such systems produce a spectral data cube - a spectrum at every $2 \mathrm{D}$ spatial position. In early systems a sequence of spatial images was acquired by means of an NIR camera and a set of band-pass filters [18], [19], [20], [21]. While the acquisition can be fast, the disadvantage of this approach is that only a limited number of wavelengths can be analysed and that they need to be known a priori. In a more recent approach a line of spatial information with a full spectral range per spatial pixel is captured sequentially to complete a volume of spatial-spectral data [18], [19], [20], [21]. This is usually achieved by means of a spectrograph which disperses an incoming line of radiation into a spectral and spatial matrix which is captured by the camera [14]. The horizontal and vertical pixels on the camera capture spatial and spectral information, respectively. Such a system provides full spectral information at every spatial position. The object must be moved stepwise under the camera by means of an actuator while at each step a line is scanned, but this is not necessarily a disadvantage when the system is mounted on a grading line on which the fruit is physically transported anyway. Novel developments include focal plane array cameras in combination with LCTF, AOTF or other monochromator principles which allow for much faster acquisition speeds [14], [18], [19], [20], [21].

\section{Chemometrics}

$\nabla$

The NIR spectrum is essentially composed of a large set of overtones and combination bands. This, in combination with the complex chemical composition of natural plant products causes the NIR spectrum to be highly convoluted [4], [13]. Additionally, the spectrum might further be complicated by wavelength dependent scattering effects, tissue heterogeneities, instrumental noise, ambient effects and other sources of variability [4], [9], [13]. As a consequence, it is difficult to assign specific absorption bands to specific functional groups let alone chemical components [4], [9].

The combination of NIR spectroscopy and multivariate techniques such as partial least squares (PLS) and principal component (PCR) regressions provides a powerful tool for the interpretation and analysis of the spectra [6], [8], [22]. Multivariate statistical techniques applied to chemical data (also called chemometrics) are required to extract the information about compositional attributes which is hidden in the NIR spectrum (model calibration) [6], [8], [22], [23].

Chemometrics covers quite a broad range of methods including exploratory data analysis, pattern recognition, and statistical ex- 
perimental design [22], [23]. The most commonly used multivariate data analysis techniques applied to NIR spectra are principal component analysis (PCA), PLS, PCR, discriminant analysis (DA) and artificial neural network (ANN) [22], [23], [24]. Chemometrics, unlike classic statistics, considers multiple variables simultaneously and takes collinearity (the variation in one variable, or a group of variables, in terms of co-variation with other variables) into account [22]. Calibration development can mathematically describe the co-variation (degree of association) between variables, or can find a mathematical function (regression model), by which the values of the dependent variables are calculated from values of the measured (independent) variables [22], [23], [24], [25]. However, when applying any of the data modelling techniques presented above, it is important to select an optimum number of variables or components. If too many are used, too much redundancy in the X-variables (wavelengths) is used and the solution can become overfitted - the model will be very dependent on the dataset and will give poor prediction results [22], [23], [24], [25]. On the other hand, using too few components will cause underfitting and the model will not be large enough to capture the variability in the data [22], [23], [24], [25]. This fitting effect is strongly dependent on the number of samples used to develop the model, and in general, more samples give rise to more accurate predictions [22], [23], [24], [25]. Typical NIR procedures to develop a calibration start from the collection of NIR spectra (reflectance or transmittance), over the $1100-2500 \mathrm{~nm}$ spectral region, of a suitable number of samples with known chemical values (e.g., analysed by analytical methods). Both spectral and analytical data are usually inspected using PCA in order to visualise outliers or atypical samples, as well as to inspect the structure of the data set. Calibration models are produced using either multiple linear regression (MLR), PLS or PCR. Data pre-treatment using mathematical transformation (e.g., derivatives, multiple scatter correction, smoothing) of the NIR spectra can be applied to enhance spectral features and/or remove or reduce unwanted sources of variation (e.g., particle size, temperature) during the development of the calibration models. After the calibration, a validation set (samples not included in the calibration) is used to test the robustness and accuracy of the model. Several statistics are used to test the accuracy and precision of the calibration models developed such as the standard error of cross validation (SECV), the standard error prediction (SEP), the coefficient of determination $\left(\mathrm{R}^{2}\right)$ [22], [23], [24], [25]. Detailed information about algorithms, sample selection and statistics used to interpret the NIR calibration and validation models can be found in specific reviews or books specialised in chemometrics [22], [23], [24], [25].

\section{Data Pre-Processing \\ $\nabla$}

In many applications the NIR spectrum needs to be pre-processed before it can be analysed using chemometric techniques. Several pre-processing techniques are available such as derivatives (e. g., first, second and third derivative), multiplicative scatter correction (MSC), baseline correction, and smoothing are utilised to correct the scattering effects, to eliminate the baseline shift, and to enhance spectral features [9], [10], [12], [22], [23], [24].

\section{Sample Preparation}

\section{$\nabla$}

One of the most frequently cited benefits of analysis by NIR spectroscopy is that little or no sample preparation prior to analysis is required [10], [26]. However, in NIR spectroscopy some sort of sample preparation is needed in order to present the sample to the spectrophotometer and to analyse it. Different factors such as particle size, moisture level, temperature, as well as physical effects (e.g., comparing whole, ground or powder samples) can affect the method of collecting the NIR spectra (e.g., reflectance vs. transmittance) as well as the calibration results [10], [26].

\section{Applications: Quantitative analysis}

Both quantitative and qualitative applications of NIR spectroscopy have been reported in the literature for analysis of natural, medicinal and aromatic plants. A summary of recent applications on the quantitative analysis of different compounds in natural products, medicinal and aromatic plants is described in the following section.

\section{Alkaloids}

Coffee is one of the most important raw materials within the international trade, for which the highest quality is demanded [2], [27]. Due to the high number of samples to be analysed, new analytical techniques providing fast and reliable data about the quality are essential [2], [27], [28]. Therefore, the development of new analytical methods based on NIR spectroscopy for the quantitation of the main compounds in coffee is of importance for the industry. The determination of three main alkaloids in roasted coffee, caffeine, theobromine and theophylline, using PLS models based on NIR spectroscopy and HPLC was reported [28]. The standard error of prediction (SEP) values obtained were $0.40 \mathrm{~g} / 100 \mathrm{~g}$ for caffeine (concentration range 0.95 to $4.13 \mathrm{~g} / 100 \mathrm{~g}$ ) and $0.10 \mathrm{~g} / 100 \mathrm{~g}$ (concentration range 0.10 and $0.67 \mathrm{~g} / 100 \mathrm{~g}$ ) for theobromine [28].

Berberine and total alkaloids content were analysed simultaneously by NIR spectroscopy and HPLC to differentiate species of Cortex Phellodendri, Cortex Phellodendri Chinensis and Cortex Phellodendri Amurensis [29]. The root mean square error of prediction (RMSEP) obtained using PLS after pre-processing optimisation (multiple scatter correction and second derivative) were $2.474 \mathrm{mg} / \mathrm{g}$ for berberine (concentration range 6.83 to $67.24 \mathrm{mg} /$ g) and $2.438 \mathrm{mg} / \mathrm{g}$ for total alkaloid content (concentration range 13.77 to $67.24 \mathrm{mg} / \mathrm{g}$ ), respectively [29]. The same authors reported the influences of water absorption on both the NIR spectra and the performance of the calibration models obtained, in order to explore the practicability of the NIR method in routine analysis [29]. Similarly, NIR spectroscopy was evaluated to determine the contents of berberine, palmatine, jatrorrhizine and total alkaloids of Coptis extracts [30]. The RMSEP values obtained for the calibrations between the NIR and HPLC data using PLS regression were $0.184,0.109,0.054$ and 0.325 for berberine, palmatine, jatrorrhizine and total alkaloids, respectively [30].

\section{Tocopherols}

The use of NIR spectroscopy to measure vitamin $\mathrm{E}$ ( $\alpha$ - and $\beta-\gamma-$ tocopherol) in fresh and dehydrated leaves of lucerne (Medicago sativa) was reported [31]. The range of vitamin $\mathrm{E}$ in the set of samples analysed was $0.55-5.16 \mathrm{mg} / 100 \mathrm{~g}$ for $\alpha$-tocopherol and $0.07-0.48 \mathrm{mg} / 100 \mathrm{~g}$ for $\beta-\gamma$-tocopherol. The SEP values 
corrected by bias obtained between the NIR and HPLC data using modified PLS (MPLS) were $0.321 \mathrm{mg} / 100 \mathrm{~g}$ for $\alpha$-tocopherol and $0.022 \mathrm{mg} / 100 \mathrm{~g}$ for $\beta-+\gamma$-tocopherol, respectively [31]. A novel aspect of this application was that a fibre optic probe attached to an NIR spectrophotometer was used to measure vitamin $\mathrm{E}$ in fresh leaf samples ( $\bullet$ Table 1 ).

The determination of $\alpha$-tocopherol in several vegetable oils (sunflower, soybean, corn, rapeseed and vegetable oil) using different wavelengths in the NIR domain was reported. The SEP values obtained between the NIR and HPLC data ranged from 0.20 to 0.37 using PLS as a regression method [32].

\section{Phenolic compounds}

Several authors reported the feasibility of NIR spectroscopy to determine total polyphenols, epigallocatechin gallate, epicatechin and caffeine in green tea and other natural products (๑ Table 2) [33], [34], [35], [36], [37].

The combination of NIR spectroscopy with PLS regression was reported to estimate epigallocatechin gallate, epicatechin and trolox equivalent antioxidant capacity (TEAC) in green tea (Camelia sinensis L.) leaves [33]. The RMSEP values for TEAC range from 88 to 175 using whole and ground leaves, respectively. The RMSEP values obtained for whole leaves range from 0.54 to 0.80 for epigallocatechin gallate and 0.069 to 0.084 for epicatechin [33].

Calibration models between NIR data and total phenolics in green tea, measured using the Folin-Ciocalteu assay were developed using three different algorithms namely PLS, interval PLS (iPLS) and synergy interval PLS (siPLS) [34]. The root mean square of cross validation (RMSECV) obtained was $0.75 \mathrm{~g} / \mathrm{g}$ (concentration range in validation 15.84 to $24.39 \mathrm{~g} / \mathrm{g}$ ) for total phenolics [34].

Calibrations for total polyphenols in green tea were developed using PLS regression applied to NIR and HPLC data. The RMSEP values obtained varied from 0.0836 to $0.1643 \%$ using different pre-processing spectral methods [35].

The determination of the total polyphenols in four varieties of green tea was reported by comparing NIR spectroscopy and

Table 1 Analysis of glucosinolates, tocopherols and kavain in several natural plant materials by near infrared spectroscopy.

\begin{tabular}{|c|c|c|c|c|c|c|c|c|}
\hline Reference & Compound & $\mathbf{N}$ & $\begin{array}{l}\text { Plant } \\
\text { material }\end{array}$ & $\begin{array}{l}\text { Wavelength } \\
\text { range }(\mathrm{nm})\end{array}$ & Range & $\begin{array}{l}\text { Algo- } \\
\text { rithm }\end{array}$ & $\begin{array}{l}\text { SECV/ } \\
\text { SEP }\end{array}$ & RPD \\
\hline \multirow[t]{3}{*}{$\begin{array}{l}\text { Font et al. } 2004 \\
\text { [49] }\end{array}$} & $\begin{array}{l}\text { Sinigrin } \\
\left(\mu \mathrm{mol} \mathrm{g}{ }^{-1} \mathrm{dw}\right)\end{array}$ & 69 & $\begin{array}{l}\text { Seeds Brassica } \\
\text { juncea }\end{array}$ & $400-2500$ & $4.7-178.2$ & MPLS & 18.74 & 2.59 \\
\hline & $\begin{array}{l}\text { Gluconapin } \\
\left(\mu \mathrm{mol} \mathrm{g}{ }^{-1} \mathrm{dw}\right)\end{array}$ & & & & $0.51-142.5$ & & 11.74 & 4.16 \\
\hline & $\begin{array}{l}\text { Total GLS } \\
\left(\mu \mathrm{mol} \mathrm{g}{ }^{-1} \mathrm{dw}\right)\end{array}$ & & & & $21.9-187.3$ & & 15.65 & 10.56 \\
\hline $\begin{array}{l}\text { Montes et al. } \\
2007[48]\end{array}$ & $\begin{array}{l}\text { Total GLS } \\
\left(\mu \mathrm{mol} \mathrm{g}{ }^{-1} \mathrm{dw}\right)\end{array}$ & 548 & Brassica napus & $960-1680$ & $4.4-21.4$ & MPLS & 2.2 & 1.3 \\
\hline \multirow[t]{2}{*}{$\begin{array}{l}\text { Gonzalez-Martin } \\
\text { et al. } 2006 \text { [31] }\end{array}$} & $\begin{array}{l}\alpha \text {-Tocopherol } \\
\left(\mathrm{mg} 100-{ }^{1}\right)\end{array}$ & 34 & $\begin{array}{l}\text { Fresh alfalfa } \\
\text { leaves }\end{array}$ & $1100-2000$ & $2.92-5.16$ & MPLS & 0.37 & - \\
\hline & $\begin{array}{l}(\beta-+\gamma \text {-)-Tocopherol } \\
\left(\mathrm{mg} 100^{-1}\right)\end{array}$ & & & & $0.21-0.48$ & & 0.027 & - \\
\hline \multirow[t]{3}{*}{$\begin{array}{l}\text { Gautz et al. } 2006 \\
\text { [45] }\end{array}$} & Kavain (\% dw) & 110 & $\begin{array}{l}\text { Dried Kava } \\
\text { (Piper } \\
\text { methysticum) }\end{array}$ & $1650-2400$ & $0.11-3.92$ & PLS & 0.21 & - \\
\hline & $\begin{array}{l}\text { Dihydro-kavain } \\
(\% \mathrm{dw})\end{array}$ & & & & $0.10-3.33$ & & 0.31 & - \\
\hline & Yangonin (\% dw) & & & & $0.08-3.02$ & & 0.47 & - \\
\hline
\end{tabular}

N: number of samples; SEP: standard error of prediction; SECV: standard error of cross validation; RPD: SD/ SEP; SD: standard deviation; GLS: glucosinolates; dw: dry weight; MPLS: modified partial least squares; PLS: partial least squares.

Table 2 Analysis of phenolic compound in several natural plant materials by near infrared spectroscopy.

\begin{tabular}{|c|c|c|c|c|c|c|c|c|}
\hline Reference & Compound & $\mathbf{N}$ & Plant material & $\begin{array}{l}\text { Wavelength } \\
\text { range }(\mathrm{nm})\end{array}$ & Range & $\begin{array}{l}\text { Algo- } \\
\text { rithm }\end{array}$ & $\begin{array}{l}\text { SECV/ } \\
\text { SEP }\end{array}$ & RPD \\
\hline \multirow[t]{3}{*}{$\begin{array}{l}\text { Manley et al. } 2006 \\
\text { [41] }\end{array}$} & 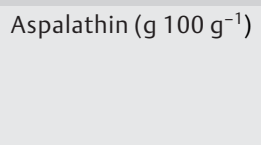 & 113 & $\begin{array}{l}\text { Dried green } \\
\text { rooibos } \\
\text { (Aspalathus } \\
\text { linearis) }\end{array}$ & $1100-2500$ & $1.13-9.79$ & PLS & 0.53 & 2.70 \\
\hline & $\begin{array}{l}\text { Dihydrochalcone } \\
\left(\mathrm{g} 100 \mathrm{~g}^{-1}\right)\end{array}$ & & & & $1.27-11.01$ & & 0.57 & 2.89 \\
\hline & $\begin{array}{l}\text { Nothofagin } \\
\left(\mathrm{g} 100 \mathrm{~g}^{-1}\right)\end{array}$ & & & & $0.10-1.21$ & & 0.10 & 2.10 \\
\hline $\begin{array}{l}\text { Luypaert et al. } 2003 \\
\text { [33] }\end{array}$ & $\begin{array}{l}\text { Epigallocatechine } \\
\text { gallate }\end{array}$ & 34 & $\begin{array}{l}\text { Leaves green } \\
\text { tea (Camelia } \\
\text { sinensis } L \text { ) }\end{array}$ & $1100-2500$ & - & PLS & $\begin{array}{l}0.29- \\
0.64\end{array}$ & - \\
\hline $\begin{array}{l}\text { Chen et al. } 2006 \\
\text { [35] }\end{array}$ & $\begin{array}{l}\text { Total polyphenols } \\
(\mathrm{g} / \mathrm{g})\end{array}$ & 20 & Green tea & $1100-2500$ & $19.38-29.17$ & PLS & $\begin{array}{l}0.0064 \\
-0.02\end{array}$ & - \\
\hline
\end{tabular}

N: number of samples; SEP: standard error of prediction; SECV: standard error of cross validation; RPD: SD/ SEP; SD: standard deviation; GLS: glucosinolates; dw: dry weight; PLS: partial least squares. 
HPLC [36]. The RMSEP obtained for the measurement of total polyphenols in ground tea leaves ranged between 1.24 to 1.79 (concentration range 19.38 to $29.17 \%$ ) using several pre-processing NIR spectra methods [36].

A quantification method to determine total phenolics content in Magnolia officinalis was established using NIR spectroscopy. Two different algorithms were used to develop the calibration models, PLS and PCR. The reported correlation coefficient (R) of calibration was above 0.95 , indicating that NIR spectroscopy was comparable to the chemical methods with regards to accuracy, prediction and reliability for practical application [38].

Wine is a complex beverage from a sensory and a chemical point of view. It can evoke a large number of olfactory responses, in addition to the taste responses of sweet, sour, and bitter and the oral sensations of astringency, irritation and viscosity. Astringency is associated with the concentration of tannins in finished wines [39], [40]. Tannins are the most abundant group of phenolic compounds typically found in red wines, and play an important role in the mouth feel properties and colour stability of wine [39], [40]. According to their chemical structure tannins can be classified as condensed or hydrolysable tannins. The use of NIR spectroscopy to predict tannin concentration and other phenolic compounds in red wines was recently reported [40].

Partial least squares was used to develop NIR calibration models to predict total polyphenols, aspalathin, nothofagin and dihydrochalcone in dried and green rooibos (Aspalathus linearis), as well as to predict aspalathin content in water extracts [41]. Results showed that NIR can effectively predict aspalathin ( $\mathrm{SEP}=0.45$, $\left.\mathrm{R}^{2}=0.85\right)$ and dihydrochalcone $\left(\mathrm{SEP}=0.49, \mathrm{R}^{2}=0.87\right)$ content of dried and green rooibos. After extending the aspalathin content range of the sample set, by adding varying amounts of dried rooibos extract powder with high concentrations of aspalathin

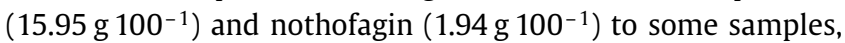
slightly less accurate, but more robust, models were obtained [41]. Excellent validation statistics were obtained for aspalathin $\left(\mathrm{SEP}=0.53, \mathrm{R}^{2}=0.87\right.$ ) and for dihoydrochalcone ( $\mathrm{SEP}=0.57$, $\left.\mathrm{R}^{2}=0.88\right)$ content, respectively. The NIR calibration model developed for nothofagin content was $\left(S E P=0.10, R^{2}=0.71\right)$ and for the extended range ( $S E P=0.10, R^{2}=0.77$ ), respectively [41].

Cyclopia genistoides normally is used for the preparation of an herbal tea, called honeybush [42]. Honeybush is a good source of the bio-active compounds mangiferin and hesperidin which are in demand for the preparation of xanthone-enriched extracts [42]. The prediction of mangiferin and hesperidin contents of the dried green plant material by NIR spectroscopy was reported [42]. The calibration sets for mangiferin and hesperidin contents ranged from 0.7 to 7.21 and $0.64-4.80 \mathrm{~g} 100^{-1}$, respectively. Using independent validation, it was shown that the PLS-NIR cali-

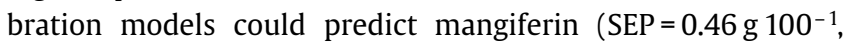

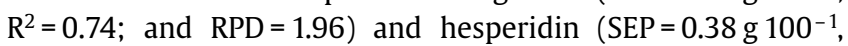
$\mathrm{R}^{2}=0.72$; and $\mathrm{RPD}=1.90$ ) contents in dried plant material, respectively [42].

NIR spectroscopy was also used to estimate quercetin content in Ginkgo biloba leaf extracts [43]. Both PLS and iPLS were used in combination with multiple scatter correction (MSC) to provide reliable PLS calibration models for quercetin [43].

Kava (Piper methysticum Forst F.), or awa in the Hawaiian language, has been used for thousands of years by the people of the South Pacific Islands, in particular Fiji, Vanuatu, Tonga, and Samoa, for social and ceremonial occasions [44], [45]. Kava has the unique ability to promote a state of relaxation without the loss of mental alertness [44], [45]. Kava recently became part of the herbal pharmacopoeia throughout the United States and Europe because of its anxiolytic properties [44], [45]. The active compounds are collectively called kavalactones (or kava pyrones). PLS calibrations were developed for kava extracts analysed using both NIR and HPLC methods [44]. The root mean square errors of calibration (RMSEC) obtained were 0.054 and 0.081 for kavain ( Table 1) and kavapyrones, respectively [44].

The combination of NIR spectroscopy and PLS has been found to be a convenient, versatile, and rapid analytical tool for determination of kavalactones in dried kava powder. Calibration equations were developed using a set of 110 samples with a diverse range in physical and chemical properties collected over time from Hawaiian kava growers and validated by analyses of a set of 12 samples with unknown kavalactones concentration [45]. All six major kavalactones and the total kavalactones were measured using NIR spectroscopy and HPLC [45]. High correlation coefficients $(R>0.99)$ and low RMSEP $(<0.094 \%)$ were obtained for the PLS models developed using NIR data [45].

\section{Glucosinolates}

The determination of glucosinolates in different plant materials (e. g., leaves and seeds) from the Brassica species by NIR spectroscopy was reported by several authors ( Table 1) [46], [47], [48], [49], [50], [51], [52], [53].

The potential of NIR spectroscopy for screening the total glucosinolates, gluconapin, gluconasturtin and neoglucobrassicin contents of cabbage leaf cultivars from Portugal and Spain was reported [46]. The SECV values were 2.60 for glucosinolates, 1.44 for gluconapin, 0.18 for gluconasturtiin and 0.21 for neoglucobrassicin $\mu \mathrm{mol} \mathrm{g}^{-1}$ dry weight (dw), respectively [46].

Optimisation of data collection processes in plant breeding programmes is of the highest importance for plant breeders when aromatic plants are improved for a particular chemical compound. For example, in rapeseed breeding, data collection and selection must be carried out in a very restricted period of time because of the short time span between harvesting and sowing [47], [48]. The use of NIR spectroscopy on combined harvesters (NOCH) improves the data collection processes in breeding programmes of grain crops such as maize, and it may also improve the data collection processes in breeding programmes of rapeseed [47], [48]. In Germany, the potential of NOCH for the determination of dry matter (DM), crude protein (CP), oil and glucosinolate (GSL) contents in rapeseed was explored [47], [48]. NOCH showed a high potential for the determination of DM, CP, oil, and GSL contents [47], [48].

Glucosinolates and their hydrolysis products are of great concern because they are responsible for many of both the beneficial and harmful properties of glucosinolate-containing plants [46], [49], [50], [51]. Cabbage (Brassica oleracea L.) is a species highly consumed as a leaf vegetable in many countries showing high qualitative and quantitative differences in glucosinolate composition among cultivars. The standard analytical techniques for determining glucosinolate composition using conventional methods lead to high costs, labour input and delays, all of which affect both the availability of data and swift decision making. A non-destructive method to screen for individual and total glucosinolates contents in 2700 winter Indian mustard seeds using NIR spectroscopy and MPLS as regression method was proposed [49]. The SEP values reported were 15.65 for glucosinolates (range from 21.89 to $187.29 \mu \mathrm{mol} \mathrm{g}^{-1}$ of $\mathrm{dw}$ ) [49]. The authors also identified that some individual glucosinolates have a specific signal in the NIR spectrum of the Brassica seed analysed [49]. 
NIR spectroscopy is widely used as an efficient and non-destructive method for seed quality analysis in oilseed rape samples (Brassica napus L.) [50]. Modified PLS calibration models were developed for NIR and HPLC data using seed samples sizes of 3-4 g [50]. The $\mathrm{R}^{2}$ and SECV reported were $0.96(\mathrm{SECV}=9.29)$ for alkenyl, $0.91(\mathrm{SECV}=1.35)$ for indole and $0.63(\mathrm{SECV}=0.34)$ for aromatic glucosinolates, respectively [50]. The use of NIR spectroscopy as a rapid method to estimate oil, fatty acids, protein and gluscosinolate contents was also reported using 2136 winter oilseed rape mutants [51].

Screening the total glucosinolate content, aliphatic glucosinolates gluconapin, glucobrassicanapin, progoitrin, glucoalyssin, and the indole glucosinolate glucobrassicin in the leaf rape (Brassica napus L. ssp.pabularia DC), was assessed using NIR spectroscopy [52]. This crop (Brassica napus L. ssp.pabularia DC) is grown for edible leaves for both fodder and human consumption, in north-western Spain (Galicia) and it is highly appreciated for human nutrition (common name of "nabicol"). A collection of 36 local populations of nabicol were analysed by NIR spectroscopy and HPLC [52]. The SECV values obtained for the individual glucosinolates ranged from 0.64 to $3.7 \mu \mathrm{mol} \mathrm{g}{ }^{-1}$ $\mathrm{dw}$. The interpretation of the PLS loadings of the first three terms of the different NIR calibration models developed, showed that some major cell components such as protein and cellulose, highly contributed in modelling the equations for glucosinolates [52].

A number of genotypes of Brassica oleracea, Brassica juncea and Raphanus sativus were analysed for individual (sinigrin, glucobrassicin, glucoerysolin, and glucocoraphenin) and total gluscosinolates over a period of 3 years using NIR spectroscopy and HPLC, using freeze dried powdered samples [53]. The RMSECV values obtained for individual and total glucosinolates range from 0.06 to 4.61 [53].

\section{Essential oils (1) Table 3 )}

Honghua oil, a traditional Chinese medicine oil preparation, is a mixture of several plant essential oils. Forty-eight commercially available Honghua oils, sourced from different batches from nine manufacturers were analysed using FT-NIR and gas chromatography (GC) [54]. Quantitative PLS calibrations were developed for three marker components, namely $\alpha$-pinene, methyl salicy- late and eugenol. The SEP values obtained were 1.55, 0.957 and 0.389 (\%) for $\alpha$-pinene, methyl salicylate and eugenol, respectively [54].

The content and composition of the volatile fraction in various pepper samples were determined by applying NIR spectroscopy and PLS regression [55], [56]. The determination of pungency, terpenoids and flavour in white and black peppercorns (Piper nigrum L) by NIR spectroscopy was reported [55], [56]. The authors concluded that efficient vibrational spectroscopy methods such as NIR and Raman, have the potential to replace the standard analysis procedures presently applied for the quality control of peppercorns, pepper extracts, and pepper oil [55], [56]. The SECV reported ranged from 0.009 to 0.27 for a wide range of essential oils analysed [55], [56].

The potential of NIR spectroscopy to assess different cinnamon (Cinnamomum zeylanicum) and clove (Syzygium aromaticum) essential oils components as well as the identification of individual essential oils was evaluated [57]. The results showed that crossvalidation models are able to predict accurately almost all of the components of essential oils. Overall, 23 components (representing 97.8 - $99.9 \%$ of the oil) were accurately predicted, as well as 20 components (93.0 - 99.1\%) in Cinnamomum camphora (ravintsara), 32 components (92.3 - 98.1\%) in Ravensara aromatica (ravensara), and 26 components (96.6 98.4\%) in Lippia multiflora [57]. For almost all of the components, the modelled and reference values obtained by GC were highly correlated $\left(R^{2} \geq 0.985\right)$ and exhibited a low variance (less than $5 \%$ ) [57].

\section{Ginsenosides}

Simultaneous quantification of ginsenosides Rb1, Rb2, Rc, Re, Rd, Rg1, Ro, m-Rb1, m-Rb2, m-Rd and m-Rc in American ginseng roots was performed by NIR spectroscopy [58]. Twenty-six samples of American ginseng roots were analysed for their content in ginsenosides using both HPLC and NIR spectroscopy. Among the calibration equations for the 11 individual ginsenosides, those of ginsenosides Rb1, Re and m-Rb1 showed the lowest relative standard deviation. These results showed that the accuracy and precision of NIR as a method for the quantification of ginsenosides is comparable with those obtained with HPLC [58]. According to the authors, the main drawback of this technique is

Table 3 Analysis of essential oils and lignin glycosides in several natural plant materials by near infrared spectroscopy.

\begin{tabular}{|c|c|c|c|c|c|c|c|c|}
\hline Reference & Compound & $\mathbf{N}$ & Plant material & $\begin{array}{l}\text { Wavelength } \\
\text { range }(\mathrm{nm})\end{array}$ & Range & $\begin{array}{l}\text { Algo- } \\
\text { rithm }\end{array}$ & $\begin{array}{l}\text { SECV/ } \\
\text { SEP }\end{array}$ & RPD \\
\hline \multirow[t]{3}{*}{$\begin{array}{l}\text { Schulz et al. } 2005 \\
\text { [55] }\end{array}$} & Piperine $(\mathrm{mg} / 100 \mathrm{~g})$ & 51 & $\begin{array}{l}\text { Black and } \\
\text { white pepper }\end{array}$ & $1100-2500$ & $2.75-5.07$ & PLS & 0.21 & - \\
\hline & $\begin{array}{l}\text { Essential oil } \\
(\mathrm{mL} / 100 \mathrm{~g})\end{array}$ & & & & $0.80-3.60$ & & 0.27 & - \\
\hline & $\begin{array}{l}\text { Limonene } \\
(\mathrm{mL} / 100 \mathrm{~g})\end{array}$ & & & & $0.01-0.52$ & & 0.04 & - \\
\hline \multirow[t]{3}{*}{ Wu et al. 2008 [54] } & $\alpha$-Pinene (\%) & 12 & Honghua oil & $1100-2500$ & & PLS & 1.55 & - \\
\hline & Methyl salicylate (\%) & & & & & & 0.97 & - \\
\hline & Eugenol (\%) & & & & & & 0.38 & - \\
\hline \multirow[t]{2}{*}{ Kim et al. 2007 [61] } & $\begin{array}{l}\text { Sesaminol } \\
\text { triglucoside } \\
(\mathrm{mg} / 100 \mathrm{~g})\end{array}$ & 30 & Sesame seeds & $400-2500$ & $7.4-128$ & MPLS & 12.7 & 2.47 \\
\hline & $\begin{array}{l}\text { Sesaminol } \\
\text { diglucoside } \\
(\mathrm{mg} / 100 \mathrm{~g})\end{array}$ & & & & $1.1-51$ & & 3.38 & 2.37 \\
\hline
\end{tabular}


the calibration step, which requires analysis of several samples covering all the expected spectral variability of the sample and a reliable HPLC method for the determination of ginsenosides as a reference method [58], [59].

\section{Glycoside compounds (C Table 3)}

The combination of visible (VIS) and NIR spectroscopy was explored as a rapid, simple and low cost measurement of the concentration of total glycosylated (G-G) compounds in white grape juice samples. PLS regression resulted in an $R^{2}$ and SECV of 0.82 and $49.15 \mu \mathrm{M}$, respectively. The results show that VIS-NIR spectroscopy has potential for use as a rapid, semi-quantitative technique to predict G-G concentration in white grape juices as 'low', 'medium' or 'high' [60]. O Fig. 1 shows the relationships between the reference method and the predicted results for $\mathrm{G}-\mathrm{G}$ using the NIR wavelength region $(900-2500 \mathrm{~nm})$.

Determination of lignans and non-lignan glycosides in intact sesame seeds (Sesamum indicum L) using NIR spectroscopy and HPLC was reported [61]. The SECV reported using MPLS as a calibration method were 12.7 for sesaminol triglucoside, 1.08 for sesaminol-(1-2)-diglucoside, 4.57 for sesamolinol diglucoside and 3.38 for sesaminol-(1-6)-diglucisode [61].

NIR spectroscopy was reported to estimate the content of the five main crocetin glycosides, the compounds responsible for colour and bitterness in saffron, on well defined samples from leading producer countries [62]. Calibration equations were developed between NIR and HPLC data using principal component regression (PCR) [62]. The SEP values reported were 1143.03 for trans-crocetin di-( $\beta$-D-gentibiosyl) ester, 204.31 for cis-crocetin di-( $\beta$-D-gentibiosyl) ester, 688.6 for trans-crocetin di- $(\beta$-D-glucosyl)-( $\beta$-o-gentibiosyl) ester, 102.1 for cis-crocetin di-( $\beta$-D-glucosyl)-( $\beta$-D-gentibiosyl) ester and 112.1 for trans-crocetin-di-( $\beta$-Dglucosyl) [62].

The effect of drying conditions on harpagoside retention, as well as the use of NIR spectroscopy for rapid quantification of the iridoids, harpagoside, 8-p-coumaroylharpagide (8pCHG) and moisture, in dried roots of Harpagophytum procumbens (devil's claw) was reported [63]. The harpagoside and 8pCHG calibration models established for both iridoid glucosides, indicated that they can be used for screening purposes to provide a semi-quan-

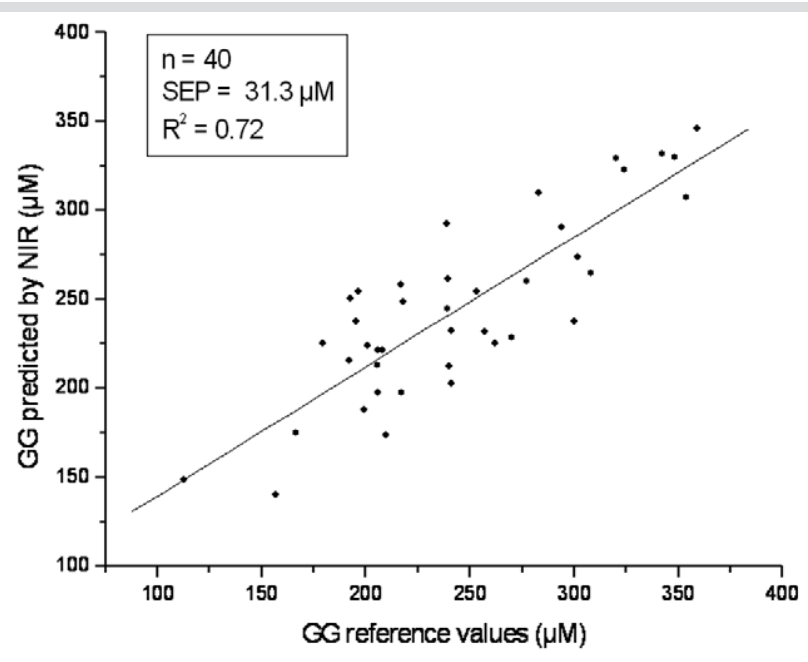

Fig. 1 Relationships between the reference method and the predicted results for G-G using the NIR wavelength region. titative classification of devil's claw roots, for harpagoside $(\mathrm{SEP}=0.236 \%, \mathrm{R}=0.64)$ and for $8 \mathrm{pCHG}(\mathrm{SEP}=0.048 \%, \mathrm{R}=0.73)$, respectively [63].

Tartary buckwheat [Fagopyrum tataricum (L.) Gaench] is rich in rutin and D-chiro-inositol, which have beneficial effects in the treatment of haemorrhagic diseases and insulin-resistant diseases, respectively [64]. The current methods of extraction and detection of rutin and D-chiro-inositol are complex and time-consuming; therefore NIR spectroscopy was explored as a method to determine the contents of rutin and D-chiro-inositol in tartary buckwheat [64]. The NIR spectral data were compared with those determined by HPLC. Models for predicting rutin and Dchiro-inositol contents in buckwheat were developed using a PLS algorithm. Cross-validation procedures indicated good correlations between HPLC data and NIR predicted values $\left(R^{2}=0.76\right)$ for rutin and $\left(R^{2}=0.86\right)$ for $D$-chiro-inositol, respectively. The rutin content ranged from 0.998 to $1.75 \%$, while the D-chiro-inositol content ranged from 0.179 to $0.200 \%$ [64].

\section{Volatile compounds}

The use of NIR spectroscopy as an alternative method to predict volatile compounds related to aroma in Riesling wines was also reported [65]. The measurement of volatile compounds using GC-MS was compared with the predictions developed using PLS and NIR spectroscopy. The SECV obtained varied from $314 \mu \mathrm{L}^{-1}$ for fatty acids, $21 \mu \mathrm{L}^{-1}$ for monoterpene alcohols and $16.6 \mu \mathrm{L}^{-1}$ for short-chain fatty acids, respectively [65].

The determination of carnosic acid in rosemary leaves (Rosemarinus offinicalis) using NIR spectroscopy was reported [66]. In this study, the authors compared three different spectrophotometers and the effect of three different temperatures (room, cool and warm temperature) to measure carnosic acid [66]. The SECV values obtained varied from 0.5 to 1.5 using three different spectrophotometers [66].

\section{Applications: Qualitative Analysis}

Several reports have been found on the classification, discrimination or authentication of several natural products by means of NIR spectroscopy and chemometrics [67], [68]. Both classification of the origin of natural products and verification of authenticity have become two of the potential applications of NIR spectroscopy.

Adulteration has been practiced since ancient times but has become more sophisticated in the recent past. Ingredients most likely to be targets for adulteration include those, which are of high-value, or are subject to the vagaries of weather during their growth or harvesting. The practice of adulteration commonly arises for two main reasons, firstly it can be profitable, and secondly adulterants can be easily mixed and are subsequently difficult to detect. To counter this problem manufacturers subject their raw material and by-products to a series of quality controls which includes HPLC, thin layer chromatography (TLC), enzymatic tests and physical tests, to establish their authenticity and hence guarantee the quality of the products manufactured [68].

A method to classify tea plant varieties based on VIS and NIR spectroscopy was developed [69]. Two hundred and ninetythree samples from field experiments were collected from three different tea gardens, and analysed using VIS-NIR. Wavelet transformation (WT), principal component analysis (PCA) and 
artificial neural network (ANN) were used to classify the tea samples. The ANN models developed gave good classification accuracy up to $77.3 \%$ for the three varieties analysed [69].

The rapid identification of green, black and Oolong teas using NIR spectroscopy and support vector machine (SVM) as pattern recognition method was reported [70]. The spectral features of each category can be used to differentiate in the NIR region the three varieties of tea analysed [70]. The best classification rates were up to $90 \%, 100 \%$ and $93.33 \%$, using the calibration set and $90 \%, 100 \%$ and $95 \%$ using the validation set, respectively [70]. The discrimination between Arabica and Robusta pure coffee varieties and blends of varied varietal composition was also achieved using NIR spectroscopy [71]. Direct orthogonal signal correction (DOSC) as a pre-processing method was applied to the NIR spectra of 191 roasted coffee samples sourced from both pure varieties and blends [71].

Licorice, the roots of Glycyrrhizia uralensis Fisch, is used as a medicinal herb and food additive in China [72]. At present, the quality grading and authentication of licorice products is generally done by visual inspection, which is subjective and often biased. A rapid and non-destructive method for the classification of licorice samples according to their growing conditions, geographic areas and plant parts was developed using a NIR spectrophotometer coupled with a fibre optic [72]. Spectral NIR differences between licorice samples were enhanced using pattern recognition techniques even though the raw spectra were very similar. The results showed that the NIR spectra of the samples were moderately clustered, and a pattern recognition technique such as soft independent modelling of class analogies (SIMCA) provided satisfactory classification results [72].

A study reported the use of NIR spectroscopy to classify essential oils sourced from cinnamon (Cinnamomum zeylanicum) and clove (Syzygium aromaticum) plant materials [57]. The NIR model developed was able to discriminate between two essential oils ravintsara and ravensara. This study demonstrated the suitability of NIR technology as a quality control tool for the rapid identification of individual essential oils, for product authentication, and for the detection of adulteration [57].

The main psychoactive component of drug type cannabis, tetrahydrocannabinol (THC) is present at low levels in hemp, which is often used in the food and textile industry. NIR spectroscopy was used to discriminate between THC-rich and hemp forms of cannabis [73].

The rapid identification of four tea varieties from four China regions namely Longjing, Biluochun, Qihong and Tieguanyin was evaluated using NIR spectroscopy and pattern recognition techniques [36]. The best classification results were achieved for Longjing (90\% in calibration) and for Biluochun ( $80 \%$ in validation).

NIR spectroscopy has been used to analyse saffron spice in order to determine the chemical composition and geographical origin of 111 samples from the there main producing countries: Iran, Greece, and Spain [62]. The NIR results were compared to those obtained by UV-VIS and HPLC measurements, demonstrating that NIR spectroscopy is appropriate to determine moisture and volatile content and other important parameters (e.g., colouring strength) established on the ISO 3632 Technical Specification Normative, as well as to certify saffron quality in the international market [62].

The discrimination of Ganoderma lucidum from different geographical growing regions in China was attempted by using NIR spectroscopy, PCA and discriminant analysis (DA) [74]. The au- thors showed that excellent classification rates (100\%) can be obtained using different spectral pre-treatments [74].

Recently, the use of NIR spectroscopy as a process analytical tool was reported in order to classify Ginkgo biloba extracts from different batches over a period of 4 years, covering different processes and industrial problems such as granulation and compression [75]. The authors concluded that the combination of pattern recognition techniques and NIR can be an excellent tool for the identification of industrial problems during the processing of Ginkgo biloba extracts [75].

Fourier transform near-infrared (FT-NIR) was applied to the online measurement of green tea composition as well as to predict tea ranking [76]. FT-NIR combined with both PCA and PLS techniques, was employed to measure changes in green tea metabolites during manufacturing and processing [76]. It was indicated by the authors that the wavenumber regions between 1800 to $1900 \mathrm{~nm}$ (O-H overtones) had high correlation with the quality of the tea. In this study, a reliable qualitative prediction model of green tea has been achieved [76].

Commercial tea samples purchased from local supermarkets in China were analysed using VIS-NIR spectroscopy [77]. Classification of samples according to variety was attempted using ANN after WT pre-processing [77]. The classification rates reported by the authors were $100 \%$ for all the eight varieties analysed [77].

The use of NIR spectroscopy was reported to discriminate between different cocoa genetic groups from different producer countries [78]. Both PCA and DA were able to classify cocoa cultivars with a rate of $89 \%$ [78]. $\odot$ Fig. 2 shows the PCA score plot (PC1 and PC2) of tea samples sourced from 5 different geographical regions analysed using NIR $(700-2500 \mathrm{~nm})$.

\section{Advantages and Limitations \\ $\nabla$}

NIR spectroscopy has been successfully applied for natural products composition analysis, product quality assessment and in production control. The NIR spectrum can give a global signature of composition (fingerprint) which, with the application of chemometric techniques (e.g., principal component analysis and partial least squares regression), can be used to elucidate particular compositional characteristics not easily detected by traditional targeted chemical analysis.

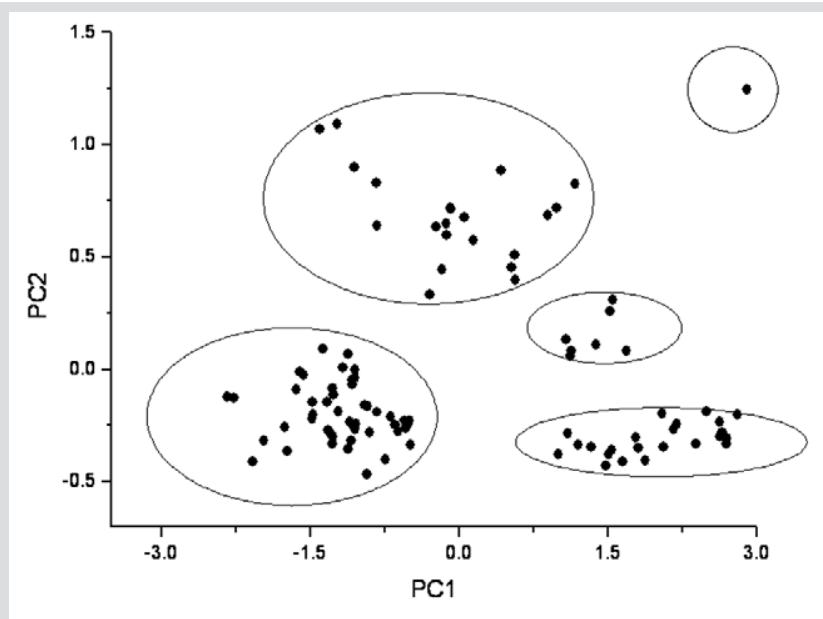

Fig. 2 Principal component score plot (PC1 and PC2) of tea samples sourced from 5 different geographical regions analysed using NIR. 
The main advantages of this technique over the traditional chemical and chromatographic methods are the rapidity and the ease of use in routine operations. Moreover, NIR is a non-destructive technique which requires minimal or zero sample preparation. However, for quantitative analyses, the spectrophotometer has to be calibrated using a set of samples (e.g., minimum 20 - 50) with known analyte concentrations obtained by suitable reference methods (e.g., HPLC, GC).

Recent advances in instrumentation and multivariate data manipulation techniques have made possible the development of new applications of NIR spectroscopy to analyse natural plant compounds. However, the lack of formal education in both NIR spectroscopy and chemometrics is still a barrier for the widespread acceptance of this technology as a tool for the analysis of aromatic plants, natural products and essential oils.

Without doubt one of the biggest challenges in the wider use of NIR spectroscopy will be the interpretation of the complex spectra obtained. Although we devoted much time to the interpretation of the models through multivariate analysis or chemometrics, the knowledge of the fundamentals of molecular spectroscopy in natural products analysis by NIR spectroscopy is still the main barrier in order to understand the basis and functionality of the models developed.

The potential savings, reduction of analysis time and cost, the environmentally friendly nature of the technology has positioned NIR spectroscopy as a very attractive technique with a bright future in the arena of the analysis of natural products. It is clear that the breadth of NIR applications, either in routine use or under developed is showing no sign of diminishing.

\section{Acknowledgments}

\section{$\nabla$}

The author would like to thank the Editors of Planta Medica Dr. Sonja Sturm, PD. Dr. Markus Ganzera and Prof. Dr. Hermann Stuppner for the invitation to write this mini-review. Dr. Paul Smith is also acknowledged for his critical review of the manuscript.

\section{References}

1 Krüger $H$, Schulz $H$. Analytical techniques for medicinal and aromatic plants. Stewart Post Harvest Rev 2007; 3: 1-12

2 Schulz H. Analysis of coffee, tea, cocoa, tobacco, spices, medicinal and aromatic plants, and related products. In: Roberts CA, Workman J, Reeves III JB, editors. Near infrared spectroscopy in agriculture Madison: ASA, CSSA, SSSA; 2004: $345-7$

3 Hounsome N, Hounsome B, Tomos D, Edward-Jones G. Plant metabolites and nutritional quality of vegetables. J Food Sci 2008; 73: R48R65

4 McClure FW. 204 years of near infrared technology: 1800 - 2003. J Near Infrared Spectrosc 2004; 11: 487-8

5 Batten G. Plant analysis using near infrared reflectance spectroscopy: the potential and limitations. Aus J Exp Agric 1998; 38: 697 - 706

6 Blanco M, Villaroya I. NIR spectroscopy: a rapid-response analytical tool. Trends Anal Chem 2002; 21: 40 - 50

7 Deaville ER, Flinn PC. Near infrared (NIR) spectroscopy: an alternative approach for the estimation of forage quality and voluntary intake. In: Givens DI, Owen E, Axford RFE, Omedi HM, editors. Forage evaluation in ruminant nutrition. Wallingford: CABI Publishing; 2002: $301-10$

8 Miller ChE. Chemical principles of near infrared technology. In: Williams PC, Norris KH, editors. Near infrared technology in the agricultural and food industries. St. Paul: American Association of Cereal Chemist; 2001: 9-29

9 Osborne BG, Fearn T, Hindle PH. Practical near infrared spectroscopy with applications in food and beverage analysis, 2nd edition. Harlow: Longman Scientific and Technical; 1993: 227
10 Roggo Y, Chalus P, Maurer L, Lema-Martinez C, Edmond A, Jent N. A review of near infrared spectroscopy and chemometrics in pharmaceutical technologies. J Pharm Biomed Anal 2007; 44: 683-90

11 Huang $H, Y u H, X u H$, Ying $Y$. Near infrared spectroscopy for on/in-line monitoring of quality in foods and beverages: a review. J Food Eng 2008; 87: $303-13$

12 Williams PC. Implementation of near infrared technology, 2nd edition. St Paul: American Association of Cereal Chemist; 2001: 145-51

13 Cozzolino D, Cynkar W, Janik L, Dambergs RG, Gishen M. Analysis of grape and wine by near infrared spectroscopy - a review. J Near Infrared Spectrosc 2006; 14: 279-89

14 Nicolai BM, Beullens K, Bobelyn E, Peirs A, Saeys W, Theron KI. Non-destructive measurement of fruit and vegetable quality by means of NIR spectroscopy: a review. Post Harvest Biol Technol 2007; 46: 99- 108

15 Workman J Jr. Near infrared spectrophotometers. In: Roberts CA Workman J, Reeves III JB, editors. Near infrared spectroscopy in agriculture. Madison: ASA, CSSA, SSSA; 2004: 11 - 3

16 Stratis DN, Eland KL, Carter JC, Tomlinson SJ, Angel SM. Comparison of acoustic-optic and liquid crystal tunable filters for laser induced breakdown spectroscopy. Appl Spectrosc 2001; 55: 999-1004

17 Crocombe RA. MEMS technology moves process spectroscopy into a new dimension. Spectrosc Europe 2004; 3: 16 - 9

18 Roggo Y, Edmond A, Chalus P, Ulmschneider M. Infrared hyperspectral imaging for quality analysis of pharmaceutical solid forms. Anal Chim Acta 2005; 535: 79-87

19 Upchurch BL, Throop JA. Effects of storage duration on detecting water core in apples using machine vision. Trans ASAE 1994; 37: 482-6

$20 \mathrm{Kim}$ MS, Chen YR, Mehl PM. Hyperspectral reflectance and fluorescence imaging systems for food quality and safety. Trans ASAE 2001; 45: $2027-37$

21 Bearman G, Levenson R. Biological imaging spectroscopy. Available at http://trs-new.jpl.nasa.gov/dspace/handle/2014/11729

22 Naes T, Isaksson T, Fearn T, Davies T. A User-friendly guide to multivariate calibration and classification. Chichester: NIR Publications; 2002: 420

23 Adams MJ. Chemometrics in analytical spectroscopy. In: Barnett NW editor. RSC Spectroscopy monographs. Letchworth: The Royal Society of Chemistry; 1995: 216

24 Siebert KJ. Chemometrics in brewing: a review. J Am Soc Brew Chem 2001; 59: 147 - 56

25 Arvantoyannis I, Katsota MN, Psarra P, Soufleros E, Kallinthraka S. Application of quality control methods for assessing wine authenticity: Use of multivariate analysis (chemometrics). Trends Food Sci Technol 1999; 10: $321-6$

26 Murray I, Cowe I. Sample preparation. In: Roberts CA, Workman J, Reeves III JB, editors. Near infrared spectroscopy in agriculture. Madison: ASA, CSSA, SSSA; 2004: 75-85

27 Pizarro C, Esteban-Diez I, Gonzalez-Saiz JM, Forina M. Use of near-infrared spectroscopy and feature selection techniques for predicting the caffeine content and roasting color in roasted coffees. J Agric Food Chem 2007; 55: 7477-8

28 Huck CW, Guggenbichler W, Bonn GK. Analysis of caffeine, theobromine and theophylline in coffee by near infrared (NIRS) compared to high-performance liquid chromatography (HPLC) coupled with mass spectrometry. Anal Chim Acta 2005; 538: 195-203

29 Chan CO, Chu CC, Mok DKW, Chau FT. Analysis of berberine and total alkaloid content in Cortex Phellodendri by near infrared spectroscopy (NIRS) compared with high performance liquid chromatography coupled with ultra visible spectrometric detection. Anal Chim Acta 2007; 592: $121-31$

$30 \mathrm{Qu} \mathrm{HB}$, Liu Q Cheng YY. Determination of the Coptis extracts alkaloids using near infrared diffuse reflectance spectroscopy. Chin J Anal Chem 2004; 32: 477-80

31 Gonzalez-Martin I, Hernandez-Hierro JM, Bustamante-Rangel $M$, Barros-Ferreiro N. Near infrared spectroscopy (NIRS) reflectance technology for the determination of tocopherols in alfalfa. Anal Bional Chem 2006; 386: 1553-8

32 Szlyk E, Szydlowsha-Czerniak A, Kowalczyk-Marzec A. NIR determination and partial least squares regression for determination of natural alfa tocopherols in vegetable oils. J Agric Food Chem 2005; 53: 6980-7

33 Luypaert J, Zhang MH, Massart DL. Feasibility study for the use of near infrared spectroscopy in the qualitative and quantitative analysis of green tea Camellia sinensis (L). Anal Chim Acta 2003; 478: 303-12

34 Chen Q Zhao J, Liu M, Cai J, Liu J. Determination of total polyphenols content in green tea using FT-NIR spectroscopy and different PLS algorithms. J Pharm Biomed Anal 2008; 46: 568-73 
35 Chen $Q$ Zhao J, Xingyi H, Haidong Z, Liu M. Simultaneous determination of total polyphenols and caffeine contents of green tea by near infrared reflectance spectroscopy. Microchem J 2006; 83: 42 - 7

36 Chen $Q$ Z Zhao J, Haidong Z, Xynyu W. Feasibility study on qualitative and quantitative analysis in tea by near infrared spectroscopy with multivariate calibration. Anal Chim Acta 2006; 572: 7 - 84

37 Yan SH. Evaluation of the composition and sensory properties of tea using near infrared spectroscopy and principal component analysis. J Near Infrared Spectrosc 2005; 13: 313 - 5

38 Yu CY, Tong ZK, Huang HH, Zhe YQ. Quantification of phenolic compound in Magnolia officinalis by near infrared reflectance spectroscopy. J Zhejiang Forestry College 2007; 24: 544 - 9

39 Rankine B. A manual of winemaking practice for Australia and New Zealand. Sydney: Pan Macmillan Australia; 1989: 374

40 Gishen $M$, Dambergs $R G$, Cozzolino D. Grape and wine analysis - enhancing the power of spectroscopy with chemometrics. A review of some applications in the Australian wine industry. Aust J Grape Wine Res 2005; 11: 296-305

41 Manley M, Joubert E, Botha M. Quantification of the major phenolic compound, soluble solid content and total antioxidant activity of green rooibos (Aspalathus linearis) by means of near infrared spectroscopy. J Near Infrared Spectrosc 2006; 14: 213 - 22

42 Joubert E, Manley $M$, Botha $M$. Use of NIRS for quantification of mangigerin and hesperidin contents of dried honeybush (Cyclopia genistoides) plant material. J Agric Food Chem 2006; 54: 5279-83

43 Zhou X, Xiang B, Wang Z, Zhang $M$. Determination of quercetin in extracts of Ginkgo biloba L leaves by near infrared reflectance spectroscopy based on interval partial least squares (iPLS) model. Anal Lett 2007; 40: 3383 - 91

44 Gaub M, Roeseler $C H$, Roos $G$, Kovar KA. Analysis of plant extracts by NIRS: simultaneous determination of kavapyrones and water in dry extracts of Piper methysticum Forst. J Pharm Biomed Anal 2004; 36: $859-64$

45 Gautz LD, Kaufusi P, Jackson MC, Bittenbender HC, Tang CS. Determination of kavalcatones in dried kava (Piper methysticum) powder using near infrared reflectance spectroscopy and partial least squares. J Agric Food Chem 2006; 54: 6147- 52

46 Font $R$, Del Rio-Celestino M, Rosa E, Aires A, De Haro-Bailon A. Glucosinolate assessment in Brassica olerace leaves by near infrared spectroscopy. J Agric Sci 2005; 143: $65-73$

47 Montes JM, Paul C, Melchinger AE. Quality assessment of rapeseed accessions by means of near infrared spectroscopy on combine harvesters. Plant Breed 2007; 126: 329-30

48 Montes JM, Melchinger AE, Reif CJ. Novel throughput phenotyping platforms in plant genetic studies. Trends Plant Sci 2007; 12: 433-6

49 Font R, Del Rio-Celestino M, Fernandez-Martinez JM, De Haro-Bailon A. Use of near infrared spectroscopy for screening the individual and total glucosinolate contents in Indian mustard seed (Brassica juncea $\mathrm{L}$ Czern \&Coss). J Agric Food Chem 2004; 52: 3563 - 9

$50 \mathrm{Hom}$ NH, Backer HC, Mollers C. Non-destructive analysis of rapeseed quality by NIRS of small seed samples and single seeds. Euphytica 2007; 153: $27-34$

51 Olejniczak J, Adamczak M, Wojciechowski A, Feide TZ, Mollers C. Near infrared reflectance (NIRS) as a useful method for analysis of induced mutations and quantitative traits in seeds of winter oilseed rape (Brassica napus L). Prace z Zakresu Nauk Rolniczych Lesnych 2005; 98-99: $197-203$

52 Font R, Del Rio-Celestino M, Cartea E, De Haro-Bailon A. Quantification of glucosinolates in leaves of leaf rape (Brassica napus ssp pabularia) by near infrared spectroscopy. Phytochemistry 2005; 66: $175-85$

53 Quilitzsch R, Schulz H, Schütze W. Evaluation of glucosinolates in leaves and stems of various Brassica species by near infrared spectroscopy. Proceedings 12th Near Infrared Conference; Hamilton, New Zealand: 2007

$54 \mathrm{Wu}$ YW, Sun SQ Zhou Q Leung HW. Fourier transform mid infrared (MIR) and near infrared (NIR) spectroscopy for rapid quality assessment of Chinese medicine preparation Honghua oil. J Pharm Biomed Anal 2008; 46: 498-504

55 Schulz H, Barinanska M, Quilitzsch R, Schütze W, Lösing G. Characterisation of peppercorn, pepper oil and pepper oleoresin by vibrational spectroscopy methods. J Agric Food Chem 2005; 53: 3358 - 63

56 Schulz H, Quilitzsch $R$, Schütze W, Krüger $H$. Near infrared spectroscopy measurement of pungency and flavour in white and black peppercorn. Proceedings 12th Near Infrared Conference; Hamilton, New Zealand: 2007
57 Juliani HR, Kapteyn J, Jones D, Koroch AR, Wang M, Charles D. Application of near infrared spectroscopy in quality control and determination of adulteration of African essential oils. Phytochem Anal 2006; 17: $121-8$

58 Yap KYL, Chan SY, Chan YW, Lim CS. Overview on the analytical tools for quality control of natural based supplements: A case study of ginseng. Assay Drug Dev Technolo 2005; 3: 383 -9

59 Fuzzati N. Analysis methods of ginsenosides: Review. J Chromatogr B 2004; 812: $119-23$

60 Cynkar WU, Cozzolino D, Dambergs RG, Janik L, Gishen M. Effect of variety, vintage and winery on the prediction of glycosylated compounds $(G-G)$ in white grape juice by visible and near infrared spectroscopy. Aust J Grape Wine Res 2007; 13: 101 - 5

61 Kim KS, Park SH, Shim KB, Ryu SN. Use of near infrared spectroscopy for estimating lignan glycosides contents in intact sesame seeds. J Crop Sci Biotechnol 2007; 10: 185 - 92

62 Zalacain A, Ordoudi SA, Diaz-Plaza EM, Carmona M, Blazquez I, Tsimidou MZ. Near infrared spectroscopy in saffron quality control; determination of chemical composition and geographical origin. J Agric Food Chem 2005; 53: 9337 - 41

63 Joubert E, Manley M, Gray BR, Schulz H. Rapid measurement and evaluation of the effect of drying conditions on harpagosne cinetent in Harpagophytum procumbens (devils claw) root. J Agric Food Chem 2005; 53: $3493-502$

64 Yang N, Guixing R. Application of near infrared reflectance spectroscopy to the evaluation of rutin and d-chiro-inositol contents in tartary buckwheat. J Agric Food Chem 2008; 56: 761 - 4

65 Smyth H, Cozzolino D, Cynkar WU, Dambergs RG, Sefton M, Gishen M. Near infrared spectroscopy as a rapid tool to measure volatile aroma compounds in Riesling wines. Possibilities and limits. Anal Bioanal Chem 2008; 390: 1911 - 6

66 Garaude-Verdier $Y$. Use of near infrared spectroscopy to measure carnosic acid content in rosemary leaves. Proceedings 12th Near Infrared Conference; Hamilton, New Zealand: 2007

67 Brereton $R G$. Chemometrics. Data analysis for the laboratory and chemical plant. Oxford: John Wiley and Sons; 2003: 489

68 Cordella C, Moussa I, Martel AC, Sbirrazzuoli N, Lizzani-Cuvelier L. Recent developments in food characterisation and adulteration detection: technique-oriented perspective. J Agric Food Chem 2002; 50: $1751-4$

69 Xiaoli L, Yong H. Discriminating varieties of tea plant based on Vis/NIR spectral characteristics and using artificial neural networks. Biosyst Eng 2008; 99: 313-21

70 Chen $\mathrm{Q}$ Zhao J, Fang CH, Dongmei W. Feasibility study on identification of green, black and Oolong tea using near infrared reflectance spectroscopy based on support vector machines (SVM)with multivariate calibration. Spectrosc Acta Part A 2007; 66: 568 - 74

71 Esteban-Diez I, Gonzalez-Saiz JM, Saenz-Gonzales C, Pizarro C. Coffee varietal differentiation based on near infrared spectroscopy. Talanta 2007; 71: $221-9$

72 Wang L, Lee FCS, Wang X. Near infrared spectroscopy for classification of licorice (Glycyrrhizia uralensis Fisch) and prediction of the glycyrrhizic acid (GA) content. LWT 2007; 40: 83-8

73 Wilson $N$, Heinrich $M$. The use of near infrared spectroscopy to discriminate between THC-rich and hemp forms of Cannabis. Planta Med 2006; 72: DOI: 10.1055/s-2006-950060

74 Chen Y, Xie MY, Yan Y, Zhu SB, Nie SP, Li C et al. Discrimination of Ganoderma lucidum according to geographical origin with near infrared diffuse reflectance spectroscopy and pattern recognition techniques. Anal Chim Acta 2008; 618: 121 - 30

75 Rosa SS, Barata PA, Martins JM, Menezes JC. Near infrared reflectance spectroscopy as a process analytical technology tool in Ginkgo biloba extract qualification. J Pharm Biomed Anal 2008; 47: 320 - 7

76 Ikeda T, Kanaya S, Yonetani T, Kobayashi A, Fukusaki E. Prediction of Japanese green tea ranking by Fourier transform near infrared reflectance spectroscopy. J Agric Food Chem 2007; 55: 9908 - 12

$77 \mathrm{He}$ Y, Li XL, Deng XF. Discrimination of varieties of tea using near infrared spectroscopy by principal component analysis and BP model. J Food Eng 2007; 79: 1238 - 42

78 Davrieux F. Genotype characterisation of cocoa into genetic groups through caffeine and theobromine content predicted by near infrared spectroscopy. Proceedings 12th Near Infrared Conference; Hamilton, New Zealand: 2007 